\title{
Le frontispice de la Pompe funèbre du duc Charles III : pensée figurée et imaginaire matériel à la cour de Lorraine au début du XVII ${ }^{\mathrm{e}}$ siècle
}

\section{Paulette Choné}

Volume 15, numéro 1, 1988

URI : https://id.erudit.org/iderudit/1073429ar

DOI : https://doi.org/10.7202/1073429ar

\section{Aller au sommaire du numéro}

\section{Éditeur(s)}

UAAC-AAUC (University Art Association of Canada | Association d'art des universités du Canada)

\section{ISSN}

0315-9906 (imprimé)

1918-4778 (numérique)

\section{Découvrir la revue}

\section{Citer cet article}

Choné, P. (1988). Le frontispice de la Pompe funèbre du duc Charles III : pensée figurée et imaginaire matériel à la cour de Lorraine au début du XVII ${ }^{\mathrm{e}}$ siècle. RACAR : Revue d'art canadienne / Canadian Art Review, 15(1), 7-18. https://doi.org/10.7202/1073429ar

\section{Résumé de l'article}

The symbolic system of power in the Duchy of Lorraine, an independent state, consolidated itself during the long reign of Charles III (1545-1608). The prince's political actions and his virtues are celebrated in a set of 74 engravings describing the 40 days of mourning that brought together representatives from all the courts of Europe. The plates reflect the solemn attitudes of the court of France, but the main influence of the engravings can be linked to Germanic, Dutch, and Italian traditions. To find the symbolism of mourning, it is necessary to look beyond the traditional ceremonial pattern to the frontispiece of the souvenir collection and the funeral prayer of the duke. A link can be found between the rhetoric of the prayer and the means of expression in the frontispiece. Secretary Claude de La Ruelle is credited with having initiated this great collection of plates. He was probably assisted in the decorative program of the frontispiece by Father Léonard Périn, a Jesuit from the University of Pont-à-Mousson, who led the funeral prayer. La Ruelle commissioned the engravings of Frédéric Brentel, accompanied by Matthieu Merian who signed the frontispiece. Although the frontispiece has been carefully described by Pierre Marot, he did not include a detailed account of its symbolic function. Given the cultural passion for symbolism in the region to which the Duchy of Lorraine belonged and the intellectual pursuits of Jesuits combined with the "culture de la curiosité," fashionable in the French court and artistic circles alike, a more detailed reading of the iconography of the frontispiece is needed.

The similarity between the structure of the frontispiece and a marquetry cabinet can only be hypothetical. The whole may be seen as a microcosm of Lorraine's hierarchical setting, uniting the naturalistic cause and the allegorical teachings. The symbolic expression, although discreet, plays a role in accentuating the senses by bringing out a more pronounced meaning at a higher level of interpretation. The source of these figurative thoughts from the frontispiece should not be attributed to Ripa's Iconologia, but rather to the equally famous works of Valeriano, known by the learned community of Nancy. Hieroglyphica are not the only source for this work, which is linked to a culture where elegantly conceived symbols dominate the teachings and documentation of events. The most captivating characteristic is without a doubt its successful synthesis of conceptual imagery and imaginative material taken from the rarest gifts of nature.
Tous droits réservés @ C UAAC-AAUC (University Art Association of Canada | Association d'art des universités du Canada), 1988
Ce document est protégé par la loi sur le droit d'auteur. L’utilisation des services d’Érudit (y compris la reproduction) est assujettie à sa politique d'utilisation que vous pouvez consulter en ligne.

https://apropos.erudit.org/fr/usagers/politique-dutilisation/ 


\title{
Le frontispice de la Pompe funèbre du duc Charles III : pensée figurée et imaginaire matériel à la cour de Lorraine au début du XVII siècle
}

\author{
PAULETTE CHONÉ
}

Nancy, France

\section{ABSTRACT}

The symbolic system of power in the Duchy of Lorraine, an independent state, consolidated itself during the long reign of Charles III (1545-1608). The prince's political actions and his virtues are celebrated in a set of 74 engravings describing the 40 days of mourning that brought together representatives from all the courts of Europe. The plates reflect the solemn attitudes of the court of France, but the main influence of the engravings can be linked to Germanic, Dutch, and Italian traditions. To find the symbolism of mourning, it is necessary to look beyond the traditional ceremonial pattern to the frontispiece of the souvenir collection and the funeral prayer of the duke. A link can be found between the rhetoric of the prayer and the means of expression in the frontispiece. Secretary Claude de La Ruelle is credited with having initiated this great collection of plates. He was probably assisted in the decorative program of the frontispiece by Father Léonard Périn, a Jesuit from the University of Pont-à-Mousson, who led the funeral prayer. La Ruelle commissioned the engravings of Frédéric Brentel, accompanied by Matthieu Merian who signed the frontispiece. Although the frontispiece has been carefully described by Pierre Marot, he did not include a detailed account of its symbolic function.
Given the cultural passion for symbolism in the region to which the Duchy of Lorraine belonged and the intellectual pursuits of Jesuits combined with the "culture de la curiosité," fashionable in the French court and artistic circles alike, a more detailed reading of the iconography of the frontispiece is needed.

The similarity between the structure of the frontispiece and a marquetry cabinet can only be hypothetical. The whole may be seen as a microcosm of Lorraine's hierarchical setting, uniting the naturalistic cause and the allegorical teachings. The symbolic expression, although discreet, plays a role in accentuating the senses by bringing out a more pronounced meaning at a higher level of interpretation. The source of these figurative thoughts from the frontispiece should not be attributed to Ripa's Iconologia, but rather to the equally famous works of Valeriano, known by the learned community of Nancy. Hieroglyphica are not the only source for this work, which is linked to a culture where elegantly conceived symbols dominate the teachings and documentation of events. The most captivating characteristic is without a doubt its successful synthesis of conceptual imagery and imaginative material taken from the rarest gifts of naturc.
Région de passage entre deux possessions espagnoles, les Pays-Bas et la Franche-Comté, lieu d'affrontement entre les mondes germanique et français, le duché de Lorraine apparaît au xvi ${ }^{e}$ siècle comme une entité originale et complexe. Toute son histoire politique, religieuse et artistique est celle d'un pays d'entre-deux. Jusqu'à l'effondrement de la guerre de Trente Ans, la Lorraine sait s'approprier des valeurs de civilisa- tions contrastées et s'affirmer comme état indépendant et moderne dans la zone la plus fragile de l'Europe. Le long règne personnel de Charles III (1559-1608) représente une période de paix et de prospérité relatives. Certes, la Lorraine n'échappe pas aux soubresauts de la conjoncture internationale ni aux retombées des luttes religieuses françaises. Sa volonté de neutralité n'empêche pas Charles in de s'engager dans la Ligue 
catholique et, surtout après la mort du roi Henri III (1589), de revendiquer la couronne de France. L'exaltation d'une chrétienté militante, l'esprit de croisade, un mythe généalogique où se mêlent l'ascendance carolingienne et la geste du roi de Jérusalem Godefroy de Bouillon, ancêtre de la dynastie, deviennent alors les points forts de la symbolique du pouvoir.

Le pays s'ouvre à la grande politique et aux échanges internationaux, pendant que la cour de Nancy, grâce aux alliances matrimoniales avec la Toscane et la Bavière, se hausse au rang des cours européennes. A la fin du $\mathrm{XvI}^{\mathrm{e}}$ siècle, Charles III fait figure de prince bâtisseur en appelant des architectes italiens à créer la "ville-neuve " de Nancy, où s'exprime un souci avancé de rationalisation des besoins. Célébrées avec enthousiasme par les contemporains mais encore peu étudiées, les industries de luxe, dont l'implantation est voulue par le duc, s'inscrivent dans le cadre d'une économie étatisée de type mercantiliste. Tapissiers de haute lice ou de cuir doré, batteurs d'or et de cuivre, fabricants de verres et de cristaux, de draps de soie et de velours affluent à Nancy, venant d'Italie et des pays rhénans, du Brabant et des Flandres. Ils propagent une culture technique à la fois ancrée dans les ressources traditionnelles du pays (argent, cuivre, verre) et vivifiée par un imaginaire matériel que traverse la rêverie sur la magie naturelle. C'est l'époque où se multiplient les prospections locales du marbre et de l'albâtre, du jaspe et de la calcédoine, où la pratique de la sériciculture, la recherche des huîtres perlières, ne se dissocient pas de l'adhésion des princes lorrains à la "culture de la curiosité "(K. Pomian). Tel est le "monde des choses" qui forme l'arrière-plan d'un exceptionnel épanouissement artistique, dominé par les personnalités du graveur Pierre Woeiriot, de Jacques Bellange, puis de La Tour, Le Clerc et Callot ${ }^{1}$.

Vers 1600 , les affinités de la Lorraine avec les cours d'Europe centrale et d'Italie ne sont pas seulement politiques. Elles se définissent par un climat intellectuel et moral, par la participation aux valeurs esthétiques et aux codes symboliques cultivés à Munich, Ambras, Prague, Mantoue et Florence. Enfin, la Réforme tridentine, dont le centre de diffusion le plus actif est l'université de Pont-à-Mousson, fondée par Charles III en 1572, trouve dans le duché un terrain particulièrement favorable. A la mort de Charles III (1608), la Lor-

1 Aperçu bibliographique dans Michel Parisse, dir., Histoire de la Lorraine (Toulouse, 1977) et dans René Taveneaux, dir.. Hustoire de Nancy, $2^{\mathrm{e}}$ éd. (Toulouse, 1987), chapitres Iv à vi. Sur la vie artistique, voir en particulier Claude Gellée et les peintres lorrains en Italie (Académie de France à Rome, Musée des Beaux-Arts de Nancy, 1982). raine est donc une pièce importante du dispositif idéologique impérial et catholique.

Les funérailles du souverain furent l'occasion de montrer à l'Europe le rang auquel était parvenue la maison ducale, d'exhiber le système symbolique qu'elle s'était construit, de manifester l'indépendance et la prospérité du pays. Des relations détaillées, un ensemble de 74 planches gravées ont conservé le souvenir de la somptueuse pompe funèbre de Charles III $^{2}$. Le duc mourut le 14 mai 1608. Pendant trois semaines, le cercueil contenant le corps embaumé demeura exposé dans la chambre ducale. Le 9 juin, on le transporta dans la Salle Neuve du palais. Sur un lit d'honneur était étendue une effigie de cire du défunt. Pendant quarante jours, on célébra quotidiennement trois messes hautes et les vêpres des morts dans la Salle Neuve, en présence de la cour. Au dîner et au souper, les repas étaient servis devant la "feinte " du mort. Le 14 juillet, le cercueil fut transporté dans la salle funèbre aménagée dans la Galerie des Cerfs. Trois jours plus tard, un cortège de trois mille personnes l'escorta depuis le palais jusqu'à la collégiale Saint-Georges. Enfin, le 18 juillet, il fut porté avec la même solennité à l'église des Cordeliers où les ducs avaient leur sépulture.

S'il faut en croire les érudits provinciaux qui ont répété à plaisir ce "proverbe ", "le couronnement d'un empereur à Francfort, le sacre d'un roi de France à Reims, et l'enterrement d'un duc de Lorraine à Nancy " étaient les trois cérémonies les plus magnifiques que l'on pût voir en Europe ${ }^{3}$. Pierre Marot a fait justice de cette exagération en rappelant que les rites des funérailles des ducs de Lorraine n'avaient rien d'exceptionnel et que les princes lorrains suivaient une coutume générale qui s'établit à la fin du Moyen Age et s'amplifia au $\mathrm{XVI}^{\mathrm{e}}$ siècle $^{4}$.

Le plus ancien récit de l'enterrement d'un duc de Lorraine est la description par la Chronique de Lorraine du cérémonial observé à Barcelone à la mort du duc Jean, lors de l'expédition de Catalogne, en 1470. C'est dans la pompe funèbre du roi René à Angers (1481) qu'apparaissent les particularités essentielles du rituel, notamment l'exposition de la "representacion" du duc, la

2 [Claude de La Ruelle], Pompe funèbre de Charles III, duc de Lorraine. Dix grandes tables contenantes les pourtraicts des cérémonies, honneurs et pompes funèbres, faitz au corps de feu sérénissime prince Charles $3^{c}$ du nom... Ce qui est outre la pompe funèbre du convoy faict aussi lors au transport dudit corps... et figuré en 48 tables... L'ordre tenu au marcher, parmy la ville de Nanc)... à l'entrée en icelle du S. prince Henry II (Nancy, Herman de Loye, 1611).

3 Lionnois, Histoire des villes vieille et neuve de Nancy (Nancy, 1805), 183 sq.; Guerrier de Dumast, Histoire et tableau..., $2^{\mathrm{e}}$ éd. (Nancy, 1847), 47-48.

4 Pierre Marot, Recherches sur les pompes funèbres des ducs de Lorraine (Nancy et Paris, 1935). 
"feinte" ou effigie du corps du défunt, revêtue des insignes du pouvoir.

Pierre Marot a publié une précieuse relation des funérailles de René iı en $1508^{5}$; les phases du deuil et le protocole observé se retrouveront à peu près dans les pompes funèbres des successeurs du vainqueur du Téméraire. Il ne faut pas y chercher une foncière originalité par rapport aux pompes funèbres d'autres princes. On notera cependant que l'usage de servir des repas solennels à la "feinte" exposée sur un lit de parade est plus précoce en Lorraine qu'en France, où il n'a pas été pratiqué avant François $\mathrm{I}^{\mathrm{e}}{ }^{\mathrm{r}}$.

Dans le dernier quart du $\mathrm{xvI}^{\mathrm{c}}$ siècle, le cérémonial des obsèques ducales se précise et s'enrichit. Inspirée des habitudes de la cour de France, la pompe funèbre de la duchesse Claude (1575), réglée par le roi d'armes Emond du Boullay, préfigure dans ses moindres détails le déroulement de celle de Charles III.

En revanche, le principe du recueil de planches représentant les différentes phases des obsèques est à ce moment d'inspiration typiquement germanique, néerlandaise ou italienne, comme l'a montré Pierre Marot. Encore n'y a-t-il dans les cérémonies de Nancy aucun emprunt évident aux modalités décoratives diffusées par ces recueils?

Respectueuses d'un canevas traditionnel, imprégnées de gravité, les solennités funèbres n'autorisaient guère l'invention symbolique fantasque ou érudite qui caractérisait les entrées. Certes, ce n'était pas là une règle absolue; le service funèbre de Marguerite d'Autriche, reine d'Espagne, à Florence (1612) donna lieu à un grand déploiement d'allégories et d'emblèmes. Les obsèques de Charles III, telles que les dépeignent les pièces comptables ${ }^{8}$, la relation imprimée du maître des

5 Marot, Recherches, Appendice, 125-35.

6 Marot, Recherches, 21. Cf. Ernst H. Kantorowick, The King's Two Bodies: A Study in Mediaeval Political Theology (Princeton, 1957) et Ralph E. Giesy, The Royal Funeral Ceremony in Renaissance France (Genève, 1960).

7 Aux recueils cités par Pierre Marot, on ajoutera en par ticulier G. B. Strozzi, Essequie del serenissimo don Francesco Medici Gran Duca di Toscana II (Florence, 1587); Baldo Catani, La Pompa funerale Fatta dall'Ill.mo E R.mo Cardinale Montalto nelle trasportatione dell'ossa di Papa Sisto il Quinto... (Rome, 1591): quatorze pages de planches gravées par Francesco Villamena; six d'entre elles représentent des statues d'allégories avec des devises sur leurs bases, disposition que l'on retrouvera dans les planches gravées par Mérian de l'entrée à Nancy du duc Henri ir, successeur de Charlcs III. Il convient encore de citcr La Pompa Funerale fatta in Napoli nell'essequie del Cattolico Re Filippo II di Austria scritta da Ottavio Caputi de Cosenza (Naples, 1599), sans figures. Fn 1612, la pompe funèbre de Marguerite d'Autriche célébrée à Naples sera relatée par Caputi dans un ouvrage cette fois largement illustré.

8 Hippolyte Roy en a fait un premier et piètre usage dans $L a$ vie à la cour de Lorraine sous Henri II. Tableau des moeurs au Xvie cérémonies, le secrétaire Claude de La Ruelle ${ }^{9}$ et les planches gravées sous la direction de celui-ci faisaient exclusivement appel au décor héraldique et aux " pièces d'honneur ", c'est-à-dire aux marques traditionnelles de la souveraineté. En fait, toute l'invention symbolique dont on prétendit faire montre à l'occasion de ces cérémonies se condensa dans l'oraison funèbre du duc et dans le frontispice extrêmement prolixe par lequel s'ouvre le recueil de planches gravées.

siècle (Paris et Nancy, 1914). Pierre Marot l'a corrigé et complété dans les Recherches sur les pompes funèbres... Le coût de la pompe funèbre peut être apprécié ct analysé grâce au compte du Trésorier génćral (Archives départementales de Meurthe-et-Moselle, B 1308, fos 104 sq.) Marot a surtout utilisé les acquits correspondants. D'Après le registre $\mathrm{B} 1308$, la dépense pour la pompc funèbre s'éleva à 171377 francs. Encore ne s'agit-il pas d'une évaluation définitive : cette somme comprend des frais engagés pour le deuil du cardinal Charles de Lorraine, fils de Charles III, en 1607; d'autre part, elle ne correspond certainement pas au coût total de la pompe funèbrc. L'étudc détaillée de ses aspects matériels et pratiques reste à faire, notamment à travers les registres du Passage et clu cellérier de Nancy. (On connait en outre le montant des frais occasionnés par la visite des ambassadeurs venus de toute l'Furope (159 265 francs) et les paiements à divers artisans: le poticr d'étain qui confectionne le cercueil (145 f.), le brodeur (1 373 f.), la femme chargéc du gardemeubles du palais (148 f.), le tapissicr (639 f.), le pelleticr (964 f.), la lingère (15l f.), le plumassier (29 f.). L'un des architcetcs de la Ville-Neuve. Jean-Baptiste Stabili, dirigea la construction de deux chapelles ardentes à la collégiale Saint-(jeorges et aux Cordeliers (1 $006 \mathrm{f}$.); l'une était un assemblage pyramidal de couronnes ducales, l'autre ćtait forméc de " pyramides". Divers travaux décoratifs furent confićs à des verriers (237 f.), aux peintres Jean Blayer de Bariscord (360 f.) et Jacques d'Anglure (30)() f.). Jean Blayer de Bariscord, mis à contribution pour " peindre les ballustres et charpenteries de la Salle Funebrc "fut aussi chargé de peindrc "main et teste de Son Altesse ", c'est-àdire de la " feinte " (94 f.). La tâche la plus lourde dans une décoration surchargée de "pièces d'honneur " et d'armoiries revenait au héraut d'armes Jean Callot (2 190 f.), père du graveur Jacques Callot. La dépense de cire pour l'ensemble des cérémonies se montait à 4474 francs, y compris la "feinte" qui était de circ blanche. (On notera que le coût de la réception des ambassadeurs venus "plaindre le deuil " de Charles III est sans commune mosure avec les frais supportés pour l'appareil décoratif de l'enterrement. Le prestige de la cour paraît lié au faste dont elle sait entourer ses invités, plutôt qu'à la splendeur des cérémonies où elle donne une représcntation d'clle-même. A titre de comparaison, précisons que dans les années 1600 à 1607, la dépense annuclle ordinaire personnelle de Charles in, à l'exclusion de celle de ses enfants, oscille entre 180000 et 200000 francs.

9 Claude de La Ruelle, Discours des céremonies, honneurs et pompe funèbre faits à l'enterrement du très-haut, très-puissant et sérenissime prince Charles 3 du nom par la grâce de Dieu duc de Calabre, Lorraine, Bar, etc. (Nancy, Jean Savine, 1609). Voir Pierre Marot, "Contrats passés pour la gravure et l'impression des planches de la "Pompe funèbre" de Charles III, duc de Lorraine ", Gutenberg Jahrbuch (1951), 140-45; A. Ronsin, Répertoire bibliographique des livres imprimés en France au xvII" sièrle. Tome $x$. Lorraine-Trois Evêchés (Baden-Baden, 1984), 117. 
Publiée à Pont-à-Mousson dès $1608,{ }^{10}$ l'oraison funèbre de Charles III avait été prononcée par un jésuite, le Père Léonard Périn, professeur de rhétorique et d'Ecriture sainte à l'université de Pont-à-Mousson ${ }^{11}$. Elle se divise en deux parties; la première, prononcée le 18 juillet 1608 , rappelle les "Grandeurs et merites " de la vie et du règne du défunt; le lendemain, le prédicateur évoque les " merites de sa mort et honneurs qui luy ont esté faicts apres icelle ". Cette structure annonce celle du recueil de planches gravées réalisées deux ans plus tard à l'initiative de Claude de La Ruelle. L'éloge du prince correspond au frontispice décrivant les vertus du duc de Lorraine et l'opulence qu'il a introduite dans ses pays. La seconde partie, après un rappel de la piété montrée par le souverain au cours de ses dernières heures, est une longue justification des "signes extérieurs du deuil " tel que le représenteront les planches avec une extrême minutie documentaire.

Pierre Marot a supposé que l'inspirateur du frontispice de la Pompe funèbre, l'auteur de son programme symbolique et des inscriptions, pouvait être le Père Périn ${ }^{12}$. L'hypothèse est d'au-

10 Léonard Périn, s.j., Orazsons funèbres sur le trespas de feu Monseigneur tres-hault, tres-illustre el serenissime prince Charles III... Duc de Calabre, Lorraine, Bar, Gueldres E'c et de feu Monseigneur son filz, tres-illustre $\mathcal{E}^{2}$. sereniss. Prince Charles Cardinal de Lorr. du tiltre de S.Agathe, Legat du S.Siege, Evesque de Metz $\dot{*}$ de Strasbourg. Prononcées à Nancy en l'Eglise collegiate de St Georges E? en la Conventuelle des R.R.Peres Cordeliers, les 18, 19 ह 21 jours de juillet, 1608 (Pont-à-Mousson, Melchior Bernard, [1608]).

11 Sur le Père Périn, voir L'Université de Pont-à-Mousson. Histoire extraite des manuscrits du P. Nicholas Abram. Publiée par le P. Carayon (Paris, 1870), 493-506; Dom Calmet, Bibliothèque lorraine (Nancy, 1751), col. 723; Eug. Martin, L'Université de Pont-à-Mousson (1572-1768) (Paris et Nancy, 1891), 295, n. 1 et passim. Né en 1567 à Stenay dans le duché de Lorraine, Léonard Périn fit ses études au collège de Clermont et entra dans la Compagnie de Jésus à Verdun en 1589. Il traduisit notamment le manuel de civilité de l'Lniversité, Bienséance de la conversation... et les Honneurs et applaudissements... du Père Wapy et composa une vie de saint Nicolas et diverses oeuvres de controverse.

12 Marot, Recherches, 90. Ajoutons que l'imprimeur strasbourgcois collicité par Claude de La Ruelle pour le tirage de la Pompe funèbre fit à Pont-à-Mousson plusieurs tractations financic̀res dont de détail nous a échappé, à l'époque où fut signé le contrat d'impression; un acte notarié indique que "Pierre viart m(aît)re du Jeu de Paulme au Pont a mousson "lui doit la somme de 600 francs (Archives départementales de Mthe-et-Mlle, $3 \mathrm{E}$ 1)02, Minutes de Jacob Aymé, 6 juillet 1610). Le graveur Frédéric Brentel n'avait pu encore avancer beaucoup son travail, commencé en principe au début de juin (ibid., contrat du 10 mai 1610). Deux faits sont certains: Herman de Loye servit d'interprète à Brentel dès ce premier contrat; entre le 10 mai et le 6 juillet 1610, de Loye fut continuellement en relations avec des habitants de Pont-à-Mousson, notamment des marchands. Nous pouvons supposer qu'il s'y fit remettre par le Père Périn les textes français et latins devant accompagner les planches et qu'il servit de relais entre le jésuite et le graveur tant plus vraisemblable que la composition du recueil reprend fidèlement celle de l'oraison funèbre. Ajoutons qu'elle en épouse aussi l'esprit. Le discours du jésuite et le recueil illustré recourent à la même thématique de façon également simple et concrète; ils montrent avec précision, sans emphase inutile, la prospérité, la beauté, la " commodité " du duché, énumèrent les bienfaits du prince pacifique et sage; ils se tiennent à l'écart des démonstrations ampoulées, des innovations symboliques trop opaques ou recherchées ${ }^{13}$.

La seconde partie de l'oraison funèbre renferme une longue justification des cérémonies observées pendant deux mois à la cour de Nancy. Emond du Boullay, commentant la pompe funèbre de Claude de Guise, avait déjà cherché à en relier la splendeur ostentatoire à des significations humanistes et chrétiennes. Ce type de justification s'insère dans un cadre général de curiosité pour les funérailles des Anciens ou des peuples exotiques (Gyraldi, Claude Guichard, Pierre Woeiriot, Procacchi). Chez le Père Périn, il constitue l'argument essentiel de l'oraison funèbre. Les larmes, suprême louange qu'un prince puisse recevoir de ses sujets après sa mort, sont chose trop pure pour faire l'objet d'un discours. Mais chacune des particularités du deuil solonnel est longuement commentée par le prédicateur.

Les "bonnets croisés" des deuillants, l'embaumement, la salle d'honneur, le lit d'honneur, la "feinte " représentant le défunt, l'usage de lui servir des repas solennels, de porter les armes à l'église, le convoi funèbre, sont successivement pris dans un faisceau d'explications convergentes. Elles sont empruntées à l'histoire sacrée (Joseph embaumant le corps de Jacob mort en Egypte) et profane (les Romains, les Perses usaient déjà de "feintes" car l'on sait que les païens " ont fait mille

13 Sur l'orateur, le Père Abram, premier historien de l'université, a ces mots: "Aussi versé dans les connaissances humaines que dans la science des choses de Dieu, il se faisait surtout remarquer par la pureté et l'élégance avec: laquelle il parlait le latin. Toutefois sa parole était beaucoup plus facile et plus agréable lorsqu'il montait en chaire après s'être contenté de quelques moments de réflexion, que lorsqu'il avait péniblement travaillé et poli son discours. L'excès de son travail jetait une certaine obscurité sur son style "(L'Université de Pont-à-Mousson [1870], 499-500). On serait tenté d'appliquer à l'oraison funèbre de Charles in ce jugement de Jacques Hennequin à propos d'un "tombeau " oratoire composé à Pont-à-Mousson une dizaine d'années plus tard: "L'invention en est simple, l'érudition discrète et religieuse, la disposition sans recherche et l'élocution à peine fleurie " (Un "tombeau" oratoire et poétique à Pont-à-Mousson en 1619 ", dans L'Université de Pont-à-Mousson et les problèmes de son temps [Nancy, 1974], 381). L'auteur étudie la laudatio funebris prononcée par un camarade d'un étudiant défunt. A cette date, le Père Périn n'était plus professeur de rhétorique, chaire qu'il avait quittée en 1604. 
choses par bonne raison ${ }^{14}$, et associent les considérations pratiques (les quarante jours du deuil solennel permettent à ceux qui veulent s'y associer de venir de loin) et spirituelles (l'effigie du mort manifeste l'espérance chrétienne de la résurrection de la chair).

Le cérémonial, rappelé et analysé méthodiquement, est décrit comme une succession de "signes" ou d' "images", pour reprendre la terminologie employée par le Père Périn. Ainsi, par exemple, le convoi funèbre est l' "image " des pompes préparées aux saints dans le ciel; la décoration de la salle d'honneur alliant la grandeur (richesse des tapisseries de haute lice brodées d'or) à la piété (ces tapisseries empruntent leurs sujets à l'Ecriture), n'est qu'une " ombre " du paradis. Les funérailles magnifiques sont faites d'une multitude de détails dictés par la foi chrétienne dans la résurrection des corps; en outre, ce sont ces apparences visibles pompeuses et mémorables qui maintiennent les assistants dans un souvenir fidèle, moyen d'assurer beaucoup de prières aux princes, auxquelles elles sont si nécessaires.

C'est aussi l'oraison funèbre qui a soin d'établir les corrélations historiques entre le prince défunt et d'illustres souverains du passé. La soigneuse compilation permettant au Père Périn de justifier un par un les détails des obsèques dresse également autour du mort un cortège de comparaisons flatteuses, sur un mode allusif: funérailles des chefs gaulois, oraison funèbre de Théodose par saint Ambroise, dépouille embaumée de Constantin, sacre des rois de France... L'assimilation du prince mort aux héros du passé, convention habituelle de l'éloge funèbre, s'accomplit par l'intermédiaire du discours sur le cérémonial.

Les graveurs détailleront avec une scrupuleuse exactitude les particularités de la pompe funèbre comme autant de scènes figées. En-deçà du recueil illustré, le discours du jésuite montre que les phases du rituel sont d'une part les signes visibles de réalités spirituelles, d'autre part les apparences indispensables pour frapper le coeur humain " instable et oublieux".

L'initiative de la réalisation d'un grand album relatant la pompe funèbre de Charles in et l'entrée à Nancy d'Henri II, son successeur, revient au secrétaire Claude de La Ruelle. La conception et la publication du recueil lui sont entièrement dues. C'est lui qui en mai 1610, c'est-à-dire aussitôt après l'entrée d'Henri Ir à Nancy (20 avril), appela à Nancy Frédéric Brentel, "Peinctre et tailleur a l'eau forte et Bourgeois de Strasbourg ", et l'imprimeur Herman de Loye, lui aussi de Strasbourg.

14 Périn, Oraisons funebres..., 87.
Rien de semblable n'avait été gravé à Nancy auparavant; par cette publication, la cour de Lorraine rivalisait avec les grandes cours européennes, et l'enjeu politique de l'entreprise n'échappa certainement pas au grand serviteur qu'était $\mathrm{La}$ Ruelle.

Nous ne reviendrons pas sur le détail des deux contrats connus passés avec le graveur et l'imprimeur, déjà analysés par Pierre Marot ${ }^{15}$. D'autres contrats restent à découvrir qui préciseraient les modifications apportées par La Ruelle à son projet et la nature exacte de la participation des "peintres de Nancy ${ }^{16}$. Toutefois, lors du premier voyage à Nancy des Strasbourgeois, le 10 mai 1610 , le dessin du frontispice était-il préparé? Le contrat avec le graveur porte en particulier sur "six grandz pourtraictz de la pompe funebre [...] la derniere desd[i]tes pieces qui est du frontispice, les pourtraictz desquelles six pieces ont esté monstrez aud[i]t M[aît]re frederich ${ }^{17}$. S'agit-il de cuivres où le dessin a déjà été reporté sur le vernis, de calques ou de vagues "patrons " ${ }^{18}$ ? Autrement dit, quel est le rôle du graveur Matthieu Mérian dans l'invention du frontispice (Fig. 1)?

Les contrats sont muets sur le travail effectivement exécuté par Matthieu Mérian, alors âgé de 17 ans, mais il faut probablement le reconnaître dans le " garcon" qui accompagnait Frédéric Brentel à Nancy ${ }^{19}$. Le jeune graveur bâlois signe au bas du frontispice: "Mathaeus Merian fecit». C'est trop peu pour affirmer qu'il eut part à la conception de cette page, mais suffisant pour admettre qu'il ne se contenta pas d'appliquer l'eau-forte ${ }^{20}$. D'après

15 Marot, "Contrats passés pour la gravure et l'impression ", 140-45.

I6 Remarques sur le rôle de Bellange dans Nicole Walch, Die Radierungen des Jacques Bellange. Chronologie und kritischer Katalog (Munich, 1971) et Amy N. Worthen, Sue Welsh Reed. The Etchings of Jacques Bellange (Des Moines, Boston et New York, 1975-1976), 10-21.

17 Archives départementales de Mthe-et-Mlle, 3 E 1002, 10 mai 1610.

18 Le contrat n'est pas très explicite; Brentel " taillera a l'eau forte "ces six planches; il est prévu que La Ruelle fournira les planches de cuivre et fera acheter "les drogues pour le vernis et l'eau forte". La suite du contrat concerne les autres planches " que led(it) s(ieu)r de La Ruelle fera tailler par les peinctres de Nancy, que led(i)t m(aît)re frederick sera tenu aplicquer le vernis sur les lammes ou planches de Cuivre, et y donnera l'eau forte apres quelles auront esté taillees ". Nous pensons que le 10 mai, La Ruelle n'eut pas autre chose que des esquisses à présenter à Brentel. Le 6 juillet suivant, il donna à Herman de Loye "les montres et patrons en papier " des lames de cuivre " pour leur largeur et longueur "-de simples maquettes-l'imprimeur se chargeant de fournir le cuivre "battu et poly".

19 L. H. Wüthrich pense que Mérian et Brentel travaillaient ensemble sur un pied d'égalité (L. H. Wüthrich, Das druckgraphische Werk von Matthaüs Merian d. Ae. [Bâle, 1966]).

20 Wüthrich, Das druckgraphische Werk, I, 10-18. C'est aussi ce que semble indiquer la forte différence du prix payé à 
Marot, La Ruelle et le Père Périn fournirent à Mérian "les éléments de son travail "; il faudrait donc voir dans le frontispice le résultat des efforts conjugués du secrétaire des ducs Charles in et Henri in, du jésuite et du jeune dessinateurgraveur, qui devait par la suite s'intéresser tout particulièrement à la culture emblématique ${ }^{21}$.

Marot, qui a pris soin de décrire la belle ordonnance de cette page, notamment les quinze tableaux du registre inférieur, ne s'est pas arrêté à ses intentions et à son fonctionnement. Pour s'être fié à la méthode parfois fructueuse qui consiste à chercher dans Ripa " la clef " des allégories ${ }^{22}$, il est conduit à reconnaître que "nous ne trouvons pas dans l'Iconologie ou la science des emblèmes de César Ripa (éd. d'Amsterdam, 1698), la clef de ces emblèmes. Rapprochons la "bonté " du frontispice, qui tient un pélican, de celle de Ripa, mais presque tous les autres symboles sont différents ${ }^{23}$.

Le frontispice de la Pompe funèbre de Charles III, en effet, n'a pas pour source le fameux répertoire allégorique dont la première édition illustrée avait paru seulement sept ans plus tôt à Rome sous un titre le décrivant avant tout comme un ouvrage pratique à l'usage des "poc̀tes, peintres, sculpteurs, et autres ". Il n'est pas du tout certain que le livre fût connu en Lorraine avant le retour de Callot en 1621. Par ailleurs, l'élaboration symbolique chez les jésuites de Pont-à-Mousson se caractérise par son indépendance à l'égard des grands répertoires et par la "nouveauté " fièrement revendiquée de ses trouvailles, ainsi que le démontreront avec éclat les fêtes de 1623 en l'honneur des saints Ignace et François Xavier.

L'ordonnance du frontispice n'évoque guère les fortes architectures qui caractérisent les frontispices des livres de cette époque, notamment les livres sortis des presses lorraines. Néanmoins, ses

Brentel pour les six premières planches (dont fait partie le frontispice) et pour les planches " taillées par les peintres de Nancy " : 45 florins à 30 gros pièce pour les premières, 15 gros pour les secondes. D'autre part. la responsabilité de Brentel pour les planches gâtées ne s'applique qu'aux premières. Le contrat d'impression concerne au total " soixante et seize Tables taillees a l'eau forte par m(aîtr)e frederick Brentel ".

21 En 1618, Mérian grave la seconde série des emblèmes de Jacobus a Bruck (Cologne) et en 1619 les emblèmes éthiques et politiques de Zincgreff.

22 Les Recherches sur les pompes funèbres... sont de très peu postérieures au célèbre article d'Emile Mâle, "La clef des allégories peintes et sculptées aux XVII et $\mathrm{XV}^{\mathrm{e}} \mathrm{II}^{\mathrm{e}}$ siècles ", Revue des Deux Mondes (1927), repris dans L'Art religieux après le Concile de Trente (Paris, 1932). L'historien lorrain ne cède nullement au jeu " à la fois facile ct dangereux [...] de nos jours assez à la mode, le lire les tableaux à l'aide de Ripa " (Julian Gallego, Vision et symboles dans la peinture espagnole du Siècle d'Or [Paris, 1968], 39).

23 Marot, Recherches, 90, n. 1. auteurs ont certainement voulu montrer l'élévation d'un monument dans un empyrée précisé par des amoncellements de nuages. Plutôt que d'imiter une partie d'édifice, l'architecture constitue l'armature de la page, qui s'apparente à un mausolée ou à un décor éphémère généreusement couvert de compartiments peints. A la rigueur, on pourrait y retrouver l'organisation d'une façade à pignon peint, avec trois ordres superposés, couronnée d'un attique, d'une vaste table d'attente, d'un cénotaphe et de statues. Les éléments d'architecture, les panneaux peints, les "sculptures " concourent à simuler un dispositif sans grande densité plastique, mais supportant une profusion de significations fortement hiérarchisées.

Mais pourquoi ne pas évoquer à propos du frontispice de la Pompe funèbre l'un de ces cabinets d'ébénisterie d'Augsbourg ou de Nuremberg dont nous savons qu'ils étaient très prisés à la cour de Lorraine? Le "monument à tiroirs" gravé par Mérian ne repose-t-il pas sur les deux préoccupations qui gouvernent l'exécution de ces petits meubles précieux, l'application des nouvelles théories perspectives et le problème de la construction des colonnes? Chacun des tableautins est un exercice de perspective; l'ensemble se présente comme une architecture illustrant la réflexion sur les ordres, fondement de la théorie architecturale vitruvienne renouvelée par Serlio et Vignola. Mérian pouvait connaître la littérature septentrionale attachée à sa vulgarisation, le célèbre Buch von fünff Sülen de Hans Blum (Zurich, 1554), ou l'Architectura von Ausstheilung, Symetrie und Proportion der fünff Seulen... du Strasbourgeois Wendel Dietterlin (Nuremberg, 1593), amplement diffusés dans les milieux artisanaux auxquels ils étaient en principe destinés ${ }^{24}$. Par ailleurs, il ne faut pas oublier le rôle important d'un architecte de Nancy, Jean La Hiere, dans la mise au point des perspectives de la Pompe funèbre. L'esprit qui inspire le frontispice est celui d'une architecture minaturisée, interprétant avec une application scolaire le catéchisme des ordres. Son auteur tente, exactement comme pouvait le faire l'ébéniste pour le cabinet de marqueterie, de "matérialiser un "temple" dans un double sens, non seulement par son contenu encyclopédique qui cherchait à embrasser l'omnes res scribiles [...] mais aussi par sa forme ${ }^{25}$.

Il n'est pas jusqu'aux oriflammes des putti, gonflés par des vents contraires, qui n'indiquent les limites de l'illusion architecturale en accentuant

24 Sur cette littérature et son influence dans les arts appliqués, voir J. von Schlosser, La littérature artistique, rééd. fr. (Paris, 1984), 413-14 et 423.

25 Von Schlosser, La littérature, 414 
la symétrie que l'on s'attend à trouver dans un meuble ou un objet décoratif.

Dans les entrecolonnements du registre inférieur (dorique, ionique, corinthien) s'insèrent des tableautins vantant les "prospérités lorraines" (Fig. 2). Les chapiteaux composites de l'ordre supérieur, où se déploient les victoires militaires des ducs, sont ornés d'oiseaux aux ailes déployées-les aiglettes sans becs des armoires lorraines - posés sur des palmettes corinthiennes, comme si pour célébrer les hauts faits du prince et de ses devanciers, on inventait un "ordre lorrain ", couronnement de la hiérarchie des ordres.

Les entablements portent des inscriptions séparées par des compartiments décorés d'emblèmes. La table d'attente et le cénotaphe ducal s'élèvent dans un éther figuré par des nuées entassées où sont assises 28 allégories (Fig. 3). De part et d'autre de la couronne ducale, deux mains à l'index tendu sortent des nuages pour préciser leur lisibilité : à gauche sont groupées les allégories des vertus du prince, à droite celles de ses bienfaits. De chaque côté du buste de Charles inI, couronné et entouré de laurier, se dressent deux allégories féminines ${ }^{26}$. Plus guerrière avec son casque, sa cuirasse à lambrequins ornée de croix de Lorraine et d'alérions, la Gloire (Lorraine) porte une palme et une statuette de la Victoire et présente un médaillon montrant la conquête de Jérusalem par Godefroy de Bouillon. La Félicité (Bar) est vêtue d'une tunique brodée de bars héraldiques; elle tient un rameau d'olivier et un caducée entouré de deux cornes d'abondance. Elles résument allégorie morale et "territoriale ", célébration d'un fait historique et exaltation des marques héraldiques.

Retenons la parenté du frontispice avec un cabinet de marqueterie, architecture complète de petites dimensions munie de "tiroirs ", figurant les facettes d'un microcosme lorrain qui s'élèverait dans l'empyrée des mérites du prince défunt.

La hiérarchie des deux secteurs figurés (monde terrestre, monde céleste) est celle-là même qui découpe l'espace dans les retables peints à la même époque. Le modèle est transposé sans difficulté de la sphère du religieux à la sphère de la célébration du prince. Cependant, cette transposition est soigneusement limitée. L'inventaire des prospérités du pays ne peut pas et n'a pas à être traité comme une représentation sacrée. Il faut l'enfermer dans le schéma, voire dans le format d'un objet à la fois décoratif et utilitaire caractéristique des goûts et des pratiques de l'aristocratie, le cabinet de marqueterie. La perception des scènes qu'il aligne peut-elle être autre chose qu'une

26 Marot avait cru y reconnaitre Mars et Minerve. euphorie terre à terre, sinon matérialiste, bien éloignée d'une effusion spirituelle?

Cette comparaison invite à lire le frontispice à l'échelle qui est la sienne, celle d'un meuble portatif où la miniaturisation de la structure et des figures, délibérée, est elle-même signifiante. Cet artifice impose ses lois aux processus symboliques mis en oeuvre ici.

La richesse et la fécondité des duchés sont un don de Dieu, rappelle une inscription. Elles sont évoquées dans quinze petits tableaux d'une remarquable précision documentaire qui constituent un véritable panorama encyclopédique des états ducaux. Dans chacun d'eux, on a placé une ou plusieurs figures-divinités allégorisées ou allégories - assises au premier plan d'un paysage vu en perspective à partir d'un point plus ou moins élevé. Ces paysages associent parfois une cartographie sommaire à la représentation de travaux ou de divertissements. Plusieurs compositions rappellent les "natures mortes au marché " montrant des jeunes femmes assises avec leurs marchandises étalées tandis qu'à l'arrière-plan de vastes paysages abritent des scìnes de chasse ou de pêche, genre pictural mis à l'honneur entre 1550 et 1580 par le Lombard Vincenzo Campi et par les Flamands Pieter Aertsen et Joachim Beuckelaer ${ }^{27}$.

Aussi est-on frappé par la saveur populaire et rustique de la plupart de ces tableautins, qui affrontent avec sincérité la description concrète du pays et des activités de ses habitants. Ils constituent bien une nouvelle, éclatante légitimation des activités industrielles ${ }^{28}$. Même les allégories tendent vers l'expression naturaliste. Le désordre des objets éparpillés autour d'elles indique une activité florissante, fière d'exhiber ses outils et ses produits, au besoin en y ajoutant l'éventail éloquent d'une panoplie, (Opificina, Fig. 4), calquée sur le modèle de la panoplie guerrière de Mars. Les divinités champêtres adoptent ici et là des attitudes noblement familières. L'iconographie n'en est pas toujours originale; Bacchus est inspiré d'une gravure d'après Martin de Vos assez répandue, utilisée notamment dans le Theatrum vitae humanae du Messin Jean-Jacques Boissard (1596). Les figures mythologiques traditionnelles échappent au souci archéologique ou l'accommodent au climat descriptif de cette partie du frontispice. Leurs attributs tendent à être des ustensiles qui s'intègrent

27 Voir Edith Greindl, Les peintres flamands de nature morte au $x v^{\prime} I^{\circ}$ siècle (Paris, 1983).

$28 \mathrm{G}$. Rose-Villequey a montré comment le gouvernement ducal avait accentué sa main-mise sur les richesses locales, mines et salines, dans la seconde moitié du xvi ${ }^{\mathrm{e}}$ siècle. A la fin du siècle, il espérait aussi assujettir à sa politique fiscale le verre, produit de renom international: Verre et verriers de Lorraine au début des Temps Modernes (de la fin du Xv" siècle au début du xvIr siècle) (Nancy, 1970), 495. 
avec naturel dans cet univers dominé par l'économie rurale.

L'importance accordée aux ressources du soussol (salines, sources chaudes, mines d'argent, de cuivre et d'azurite, carrières de marbre et d'albâtre), aux verreries, aux huîtres perlières de la Vologne célèbre l'idée d'une nature artisane, ainsi que l'impulsion donnée par Charles II à des industries et à des prospections nouvelles. Celles-ci suscitent l'invention d'autant d'allégories inédites dont la plus belle est sans doute Volona, divinité des "perles de Lorraine " présentant des colliers devant le panorama de la ville de Bruyères, dans les Vosges (Fig. 5). Volona, en outre, célèbre métaphoriquement la nouvelle duchesse de Lorraine, Marguerite de Gonzaguc, dont le nom signifie "perle".

Au-dessus de chaque colonne, le graveur a incrusté - rejoignant ainsi l'étymologie d'emblema, "incrustation, marqueterie, mosaïque "-de minuscules compartiments décorés d' "emblèmes ", qui ont échappé à l'attention des commentateurs (Fig. 6).

Ce sont des images presque partout extrêmement schématiques, accompagnées d'un intitulé. Les quatre compartiments de l'attique, qui s'ornent de marques para-héraldiques (croix double, bras armé), de trophées et de la représentation apparente d'un lion et d'un agneau, difficilement lisible (elle ne se rencontre nulle part dans la symbolique ducale) n'ont pas de motto. Le décor des dix-huit autres compartiments fonctionne selon le processus emblématique. La figure est très simplifiée, à la limite de la lisibilité, et le motto est très bref, parfois un mot unique, comme chez Alciat. Cependant l'un et l'autre sont toujours dans un rapport de complémentarité, le motto exprime le signifié, en dehors de toute tautologie. Seule exception, le mot Insidiae qui accompagne la nasse du pêcheur n'ajoute guère à sa signification (Fig. 6). Ce sont les inscriptions, voire le contenu déclaratif des tableautins, qui assument le rôle indispensable de la subscriptio, sans laquelle on ne serait pas en présence d'emblèmes au sens strict.

Ces emblèmes discrètement encastrés dans l'architecture du frontispice n'en sont assurément pas la partie essentielle. Toutefois, ils permettent d'en lire le message descriptif et naturaliste à un niveau différent, elliptique et érudit. Si l'auteur de ce programme est bien le Père Périn, on imaginera que le professeur de rhétorique a voulu par là effectuer la "mise en style " d'un contenu que les allégories féminines n'arrachent pas suffisamment à la banalité. Cette impression est renforcée par la variété quasi-pédagogique des tropes sur lesquels reposent ces emblèmes.
Certains, par exemple, sont fondés sur des allusions savantes. Une meule de paille (Serenifer) accompagne la représentation de Palès, déesse des troupeaux. Or le mythographe Natale Conti rappelle: "Quelques Latins disent qu'elle fut ainsi nommée de palea, c'est-à-dire paille. Et de faict on celebroit certaine feste en son honneur nommée Palilia, c'est à dire feste de Palès, particuliere aux bergers " ${ }^{29}$. Le cerf blessé est symbole de guérison (Sanatio, Fig. 7) parce qu'il est réputé guérir de ses blessures en broutant l'herbe appelée dictame, ainsi que l'attestent Aristote et Pline; le motif n'a pas échappé aux emblématistes, puisqu'on le rencontre dans les recueils d'imprese de G. Ruscelli et de Simeoni et chez Camerarius. Le cerf et la chasse du cerf, mis en parallèle avec la vie du Christ, composaient toute la décoration de la Galerie des Cerfs au Palais ducal de Nancy, restaurée par Bellange en 1606; bien qu'il ne comportât aucune référence au dictame, cet ensemble de peintures et de huitains accentuait certainement la pertinence du motif inséré dans le frontispice de la Pompe funèbre ${ }^{30}$. Il est placé à l'intersection des allégories de la forêt de chênes, personnifiée par une dryade assise sur un cerf, et de la vénerie, Venatio. S'il est facile d'opérer ces rapprochements, il l'est beaucoup moins d'en saisir les intentions. L'allusion au dictame renvoie-t-elle aux plantes médicinales qui croissent dans les forêts lorraines? L'accent est-il mis sur l'animal cher à Diane, ou sur le symbolisme christique du cerf? Quel est le malum medicabile dont l'idée est sugérée? La mise entre parenthèses de la partie explicite du message est ici particulièrement excitante. L'emblème minuscule agit comme un mécanisme qui permet d' "ouvrir" la scène représentée dans le tableautin et d'en rendre plus manifestes les significations potentielles. La sanatio est en correspondance avec les vertus curatives des plantes utilisées dans leurs rites funéraires par les dryades de Luctus juventutis..., recueil de poèmes dans lesquels les élèves du collège des jésuites de Pont-à-Mousson avaient célébré le prince défunt ${ }^{31}$. L'emblème fait accéder l'allégorie à un second niveau de signification; il la

29 Natale Conti, Mythologie... (Lyon, Paul Frelon, 1607), 512. 30 Nicole Rcymaud, " La Galerie des Cerfs du Palais ducal de Nancy ", Revue de l'Art (1983), 7-28.

31 l.uctus juventutis academiae mussipontanae in funere serenissimi Caroli III (Pont-à-Mousson, Melchior Bernard, 1608). C'est le récit de funérailles bucoliques et mythologiques: les Néréides procèdent à la toilette du cadavre du duc Charles; puis les Dryades lui font un mausolée de verdure avec des arbres qui sont les signes (indices) des vertus ducales, le cyprès (bon exemple), le sapin (vaillance), le chêne (force); Flore apporte de l'hysope, Naïs du persil (protège contre les vénéfices des démons) et des violettes (dont le parfum combat la puanteur des hérétiques). 
fait entrer en résonance avec une idée qui n'est pas elle-même représentée: celle du princeguérisseur.

La chaîne symbolique est parfois plus courte, par exemple quand la chasse à l'oiseau (Aucupium) est mise en rapport avec le phénix, Unica avis, symbole de résurrection. Elle n'est pas moins énigmatique : quel est le concept reliant l'oiseau mythique et le noble plaisir de la chasse à l'oiseau, pratiquée avec passion à la cour de Charles III? Quoi qu'il en soit, on n'hésitera pas à remarquer que le " tiroir " central du frontispice, dévolu aux plaisirs aristocratiques - et meurtriers-est encadré par deux emblèmes de guérison et de résurrection.

Plus loin, le "symbolisme de la nature " cède la place à une figure de mots: deux évêques, les envoyés du Christ, sont accompagnés du " mot " sal terrae, conformément à la parole évangélique ${ }^{32}$ (Fig. 8). Le "sel de la terre " est aussi, à la lettre, le sel gemme des gisements de Rosières, Salonne, Château-Salins, Moyenvic, Marsal, Dieuze dont l'exploitation est représentée dans la vignette correspondante, Salina ${ }^{33}$. Agent de sapidité et de protection contre la corruption, le sel est projeté vers la dimension symbolique qui est la sienne dans la tradition chrétienne. Une lecture plus précise et locale de l'image des deux évêques conduirait même à y reconnaître une allusion au grand saint intercesseur lorrain, saint Nicolas. Le plus populaire de ses miracles est précisément la résurrection des trois enfants "mis au saloir", et le pélerinage qui honore le saint évêque a lieu à Saint-Nicolas-de-Port, la grande ville de foires où transite le sel lorrain des gisements tout proches ${ }^{34}$.

Le rôle de ces petits emblèmes est de stimuler les associations, d'amener le spectateur d'images au contenu assez trivial, à un niveau supérieur d'interprétation.

Nul parti-pris pédant ou moralisateur dans ces emblèmes si "effacés ", mais seulement une invitation à ne pas laisser l'oeil rivé aux phénomènes. Ainsi, la représentation de l'activité des verriers ${ }^{35}$

32 "Vous êtes le sel de la terre" (Matt. 5, 13).

33 Sur l'approche ethnologique des coutumes liées au culte de saint Nicolas, voir Collete Méchin, Saint Nicolas: fêtes et traditions populaires d'hier et d'aujourd'hui (Paris, 1978).

34 Sur l'importance du sel dans l'économie lorraine, notamment à l'époque de Charles 111, voir Le sel et son histoire. Actes du colloque de l'association interuniversitaire de l'Est (Nancy, 1979).

35 Rose-Villequey, Verre et verriers. L'auteur remarque l'extrême précision documentaire de la vignette consacrée à Vitriaria. Pour Metallica, nous avons noté un emprunt aux planches minières de la Cosmographie de Sébastien Munster (éd. de 1550), qui décrivent les mines vosgiennes du Val de Lièpvre, appartenant aux seigneurs de Ribeaupierre. Mérian copie fidèlement la représentation du mineur, du décombreur, de la fonderie; l'allégorie féminine est figurée est complétée par une métaphore illustrant la fragilité de la Fortune: Vitrea est (Fig. 9). Déjà pertinente par elle-même, l'analogie se trouve nourrie par une tradition iconographique, notamment germanique (H. Baldung Grien, Dürer, Th. de Bry) qui dote volontiers Fortune-ou Némésis-de coupes de verre qu'elle brandit et découvre comme Pandore. Elle complète aussi de son avertissement l'emblème précédent (Ubertas argentae, Fig. 10) qui présentait la corne d'abondance déversant des pièces d'argent, attribut de la Fortune positive, la FortuneRichesse. Les mines d'argent et les verrerries lorraines deviennent l'occasion d'une méditation chuchotée sur l'ambivalence de Fortune, dont les métaphores sont introduites par la "matière rêvée " autant que par la suggestion des mots.

L'emblématisation reste aimable, dense et grave, jusqu'au deux termes, Terminus (Fig. 11). L'élément de décor architectural réservé au sculpteur que célèbrent Marmorina et Alabastrina indique dans un esprit erasmien la fin de la vie terrestre et le début de la vie éternelle (Fig. 12). Le sens emblématique est donné au spectateur plus attentif; la ségrégation intellectuelle distingue celui qui sait dépasser le regard euphorique sur les realia, faire jouer la profondeur de l'expérience.

Le procédé utilisé est si discret que le message emblématique du frontispice n'a jamais été étudié. Il nous paraît fondamental parce que réduit à des signes élémentaires sertis délicatement dans la "marqueterie " qui prétend embrasser tout un règne et tout un pays, il se dérobe à une perception immédiate. En fait, il permet de révéler les significations latentes dans le "bilan économique ", la profusion empirique déployés sous nos yeux avec une sorte de sensualité entraînante. Il lui revient en outre de développer le sens symbolique des allégories, dont le rôle en principe ne va pas audelà de l'expression d'un concept. Enfin, il donne une unité supérieure à l'accumulation.

En revanche, c'est l'allégorie qui triomphe dans l'empyrée où s'élève le tombeau de Charles III. La juxtaposition peu élégante des figures assises isolées sur des nuages s'accorde avec le didactisme du propos. A deux exceptions près, ce sont des allégories féminines, encombrées d'attributs pléthoriques. L'éparpillement sans grâce des figures, l'application laborieuse avec laquelle on les a accablées d'accessoires, nuisent beaucoup à l'équilibre plastique du frontispice. L'auteur de ce programme a voulu illustrer mot à mot un discours avec son exorde (au centre) et ses deux parties (à gauche et à droite). Au-dessus du buste de

comme une "cloweresse" (trieuse de minerai) tenant la baguette de découverte. 
Charles III, trois figures allégoriques (Eternité, Gloire, Mémoire) expriment les trois premières unités lexicales (Æternæ et Gloriosæ Memoriæ) de la phrase qui décrit et explique le quart supérieur du frontispice. Celui-ci est l'énumération narrative ordonnée des vertus et des actions politiques du prince, désormais éternisées aux cieux ( $A n$ nexe).

L'abstraction figurée ne s'y dispense à aucun moment du secours du texte. La désignation est elle-même figurée par les deux mains à l'index tendu qui indiquent le bon itinéraire du déchiffrement. On n'oubliera pas que des auteurs comme Ripa et le cardinal Charles Borromée insistent sur la nécessité de renforcer l'image par la subscriptio. En outre, la redondance descriptive est le mode de fonctionnement normal et naturel des relations de fêtes et d'entrées. Dans la relation de l'entrée triomphale des archiducs Albert et Isabelle à Anvers en 1602, dont les sources explicites dont d'ailleurs très voisines de celles de la Pompe funèbre de Charles III (Valeriano, Alciat), l'ensemble du commentaire est purement descriptif, le texte redoublant exactement l'image allégorique $^{36}$.

L'effort pour ajuster les images au discours s'affirme particulièrement dans la moitié droite consacrée aux bienfaits du duc. L'allégorie s'y complique d'actions insolites qui l'apparentent à l'emblème, quand elle n'emprunte pas ses motifs à des emblèmes célèbres.

Les sources de ces allégories ne sont pas à chercher dans Ripa, nous l'avons dit. Beaucoup de figures sont fidèles à une iconographie fixée bien avant la codification de Ripa (la colonne de la Force, le miroir de la Prudence). Ripa met en effet au point une symbolique renouvelée, beaucoup plus sophistiquée, attribuant par exemple à la Prudence l'anguille et le cerf. En revanche, le répertoire de Valeriano, qui avait été largement utilisé par les auteurs nancéiens du programme de l'entrée de Marguerite de Gonzague (1606), paraît avoir inspiré ici plusieurs trouvailles. Ainsi, le choix des Grâces pour représenter les "bienfaits passés, présents et futurs " du prince, dérive certainement des Hiéroglyphiques ${ }^{37}$, qui ont également

36 Par exemple, l'allégorie de la Terreur, un homme à tête de lion, tenant un fouet, est accompagnée de cette indication : "Terror vir capito leonino personatus, flagellem manu tenens" (Johannes Boch, Historica Narratio profectionis et inaugurationis serenissimorum belgii principum Alberti et Isabellae [Anvers, J. Moretus, 1602]).

37 Commentaires hieroglyphiques ou Images des choses de Jan Pierius Valerian... (Lyon, Barthélemy Honorat, 1576), chapitre 59. I.cs trois Grâces avaient orné le revers d'une médaille de Catherine de Bourbon, la première épouse protestante du future duc Henri II; on connaît un dessin de Jacques Bellange représentant les Grâces, reproduit dans F.-G. Pariset, "Les Grâces du musée d'Alger ", Etudes d'Art (1953), 5. diffusé l'image de la "Cicogne, amie de pieté " 38 et du boeuf "fruict des labeurs" ${ }^{39}$. C'est encore Valeriano qui rappelle qu' "aucuns (suivans l'histoire Rommaine) entendent la garde par la figure de l'oye ${ }^{40}$, qui suggère de représenter la Prudence par une femme bifrons brandissant un serpent, ou qui cite la "douceur et débonnaireté " parmi les significations les plus communément reçues de l'agneau. Certes, il n'est pas question de prétendre que les Hiéroglyphiques ont été consultés pour fournir les topoi les plus fréquents, comme la cornucopia de Liberalitas $^{41}$. Valeriano brasse trop de savoirs symboliques éclectiques pour constituer une clé de lecture unique. Disons seulement qu'il était connu et pratiqué familièrement à Nancy, ainsi que le montrent les inventaires de bibliothèques ${ }^{42}$.

L'invention, dans cette partie du frontispice, se relie elle-même à l'ensemble d'une culture des symboles très diversifiée. L'Hercule féminisé qui figure Magnanimitas est à rapprocher des représentations d'Hercule personnifiant le prince magnanime ${ }^{43}$. Le motif très célèbre de l'ancre et du dauphin, transposé ici en attribut de Salus populi, provient des emblémistes qui l'affectent parfois d'une signification politique. Le prince est ami de son peuple, comme le dauphin l'est de l'homme; c'est le sens d'un emblème d'Alciat ${ }^{44}$. C'est encore d'un emblème d'Alciat qu'il convient de rapprocher la figure commentée par le motto Omnium otium ex illium labore. Le motif du putto dont une main est tirée vers le haut par une paire

38 Valeriano, Commentaires hieroglyphiques, chapitre 17. (On retrouvera fréquemment cette signification dans les emblèmes politiques du milieu du $x_{\text {vir }}{ }^{\mathrm{e}}$ siècle (I)icgo de Saavedra, Idea de un Principe politico Christiano... [Munich. 1640 ].

39 Valeriano, Commentaires hieroglyphiques, 61.

40 Valeriano, Commentaires hieroglyphiques, 443

41 Valeriano, Commentaires hieroglyphiques, chapitre 56. En revanche, Valeriano éclaire bien des détails de ces allégories. Si Clementia porte la Lune sur sa tête, et Iustitia le Soleil. c'est parce que la première est un trait éthique du législateur, tandis que la seconde est une vertu d'émanation divine; Valeriano rappelle que le Soleil est l'image de la nature divine, et que la Lune dénote la nature humaine.

42 La Bibliothèque municipale de Nancy conserve un exemplaire de l'édition bâloise des Hieroglyphica de 1567 (ex-libris Thierry Alix, Président de la Chambre des Comptes de Lorraine), un exemplaire de la traduction française de Gabriel Chappuys de 1576 (ex-libris autographe du poète Nicolas Clément de Trèles), Les Hieroglyphiques dans la traduction de J. de Montlyart (exemplaire ayant appartenu au Conseil de Ville [Lyon, 1615]), enfin une réédition latine de 1626 .

43 Voir Josè phe Jacquiot, " Hercule aux revers de médailles et

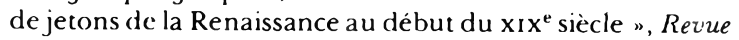
Belge de numismatique et de sigillographie (1971), 243-65; Marcel Simon, Hercule et le christianisme (Strasbourg, 1965).

44 Arthur Henkel et Albrecht Schöne, Emblemata. Handbuch zur Sinnbildkunst des XVI. und XVII. Jahrhunderts. Sonderausgabe (Stuttgart, 1978), col.683-84. L'emblème d'Alciat comporte le motto: Princeps subditorum incolumitatem procurans. 
d'ailes et l'autre liée à une lourde pierre dérive d'une illustration du Songe de Poliphile; il inspire plusieurs emblèmes de Junius, Sambucus et Rollenhagen. Alciat l'utilise pour expliquer que la pauvreté constitue un obstacle au génie ${ }^{45}$. Dans le frontispice de la Pompe funèbre, il subit une adaptation nouvelle et originale; un personnage en armure élève d'une main un leurre servant à la chasse à l'oiseau; son autre main est libre, mais il a à côté de lui un boeuf couché, qui exprime le poids du labeur. Les fatigues du gouvernement des hommes rendent possible le loisir, symptôme de paix et de prospérité publiques. Si les allégories des vertus et mérites de Charles in relèvent dans l'ensemble d'une codification appliquée, presque scolaire, leur invention témoigne néanmoins d'une familiarité acquise avec l'emblématique et d'un effort de formulation des concepts de philosophie politique qui dépasse les modes de célébration conventionnels.

On n'oubliera pas que le recueil préparé par Claude de La Ruelle, programme-souvenir offert aux invités illustres des obsèques de Charles III, était destiné à matérialiser la place à laquelle prétendait la Lorraine parmi les cours européennes ${ }^{46}$. Toutefois, il serait imprudent de réduire les

45 Viri clarissimi D. Andreae Alcati... Emblematum liber (Augsbourg, 1531), f" A 7 b.

46 Rappclons que la Pompe funèbre fut en principe tirée à 1020 exemplaires.

ANNEXE:

LISS ALLÉGORIES DE LA PARTIE SUPÉRIEURE DU FRONTISPICE (DESCRIPTION)

L'ARGUMENT (partie centrale)

\begin{tabular}{|c|c|}
\hline AETERNAE & $\begin{array}{l}\text { - une sphère armillaire posée } \\
\text { sur ses genoux }\end{array}$ \\
\hline & - tient un serpent ouroboros \\
\hline et & $\begin{array}{l}\text { - une couronne d'étoiles } \\
\text { autour de la tête }\end{array}$ \\
\hline GLORIOSAE & $\begin{array}{l}\text { - ange à la robe étoilée } \\
\text { - tient une couronne étoilée } \\
\text { resplendissante }\end{array}$ \\
\hline MEMORIAE. & $\begin{array}{l}\text { - un coffre hermétiquement } \\
\text { fermé sur ses genoux } \\
\text { - montre son front de l'index }\end{array}$ \\
\hline
\end{tabular}

LES VERTUS DU PRINCE (à gauche)

$\begin{array}{ll}\text { CHARITES } & - \text { les Grâces assises enlacées } \\ \text { MODERATIO } & - \text { tiennent des fruits } \\ \text { FORTITUDO } & - \text { tient un niveau } \\ \text { LIBERAIITAS } & - \text { tient une couronne } \\ & \text { tient une cornucopia } \\ & \text { renversée d'où s'échappent } \\ & \text { des pièces }\end{array}$

cérémonies et leur relation illustrée à la poursuite d'un effet "publicitaire". Elles recourent uniformément à un outillage figuratif assez traditionnel, à une rhétorique sereine, bénigne, sans dramatisation, sans amplification. L'empreinte intellectuelle de ses concepteurs, un familier du pouvoir et sans doute un savant jésuite, y est très sensible. L'allégorie y est employée comme un instrument commode et souple bien conforme à la morale de la communication adoptée par la Compagnie. Sertis dans l'architecture du frontispice, les éléments emblématiques fonctionnent presque comme les poussoirs secrets d'un délicat mécanisme d'ouverture d'un meuble (et du sens). Ainsi, cette page d'allure si déclarative et prolixe échappe à la codification plate et laborieuse. En déployant le tableau d'un microcosme riche des plus rares productions de la nature, en associant étroitement le prestige de la civilisation matérielle et la promotion de la pensée figurée, elle contraint l'interprète à dépasser le déchiffrement iconographique et à mettre en relation les formes symboliques avec "toute une culture en mouvement $"{ }^{47}$.

47 “Le problème primordial n'est plus celui du déchiffrement qui épuiserait la mise en relation du texte avec l'image, mais celui du sens qui oblige à mettre en relation le développement artistique avec toute une culture en mouvement" (André Chastel, Préface à la rééd. de E. Mâle,L'art religieux du XVII" siècle [Paris, 1984], 9-10)

5, Rue des Etats 54000 Nancy, France

$\begin{array}{ll}\text { MAGNANIMITAS }- & \text { tient une massue } \\ - & \text { unc dépouille de lion sur sa } \\ & \text { tête (Hercule féminisé) } \\ \text { VIRTUS HEROICA - } & \text { femme en armure, appuyée } \\ & \text { sur une lance, un pied posé } \\ & \text { sur une sphère } \\ \text { PRUDENTIA } & \text { personnage bif (jeune femme } \\ & \text { et vieillard) } \\ - & \text { brandit d'une main un } \\ & \text { serpent et de l'autre un } \\ & \text { miroir } \\ - & \text { tient un agneau contre son } \\ & \text { giron } \\ - & \text { la Lune sur sa tête } \\ - & \text { tient une épée entourée d'un } \\ \text { MANSLETLDO } & \text { rameau } \\ \text { CLEMENTIA } & \text { unc charte posée sur ses } \\ & \text { genoux: "Codicilli veniales" } \\ - & \text { le Soleil sur sa tête } \\ - & \text { tient une épée et une balance } \\ - & \text { sur ses genoux un pélican } \\ \text { IUSTITIA } & \text { nourrissant ses petits de ses } \\ & \text { entrailles }\end{array}$


- femme voilée, une flamme au-dessus de sa tête

- auprès d'elle une cigogne

- tient un livre et une croix

LES BIENFAITS DU PRINCE (à droite)

RELIGIONEM PROPAGAVIT

- femme voilée tenant une église sur ses genoux

MULTAS URBES MURO ET FOSSA COMMUNIVIT

- femme couronnée d'une tour et d'un bastion

- sur ses genoux la maquette d'une ville fortifiée

CLARAM ACADEMIAM MUSSIPONTINEM INSTITUIT, DOTAVIT FOVIT ET FLORERE FECIT

- femme tenant une palme

- trois livres (facultés des Arts, de Théologie et de Droit) sur ses genoux

COMMERCIA EREXIT

- femme assise sur une balle de marchandises

- tient une bourse pleine

HOSTIUM IMPETUS FORTITER REPRESSIT

- femme casquée, une lance à la main

- à ses pieds le bouclier orné de la tête de Méduse

EXERCITUI QUEM CONTRA HOSTES 16. ANNIS HABUIT STIPENDIUM PERSOLVIT

- femme déversant des pièces d'or sur des trophées
FOEDUS CUM FINITIMIS FIRMAVIT

- femme tenant un rameau d'olivier

- auprès d'elle un caducée et une "foi " posés sur une base carrée

QUIETEM STABILIVIT

- femme accoudée à une colonne

- tient un rameau d'olivier

OMNIUM DIVITIAE EX ILLIUS BENEFICENTIA

- femme assise entre un coffre vide et un coffre débordant de joyaux

OMNIUM SOMNUS EX ILLIUS VIGILANTIA

- femme tenant un sceptre

- sur sa tête, une grue serrant une pierre dans sa patte

- à côté d'elle, une oie

OMNIUM OTIUM EX ILLIUS LABORE

- homme en armure tenant une leurre

- près de lui, un boeuf couché

CUM SUBDITIS QUASI PARENS CUM LIBERIS

- un vieillard coiffé d'un bonnet conique

- auprès de lui, trois petits enfants nus

POPULO SALUS FUIT

- femme tenant une ancre entourée d'un dauphin 


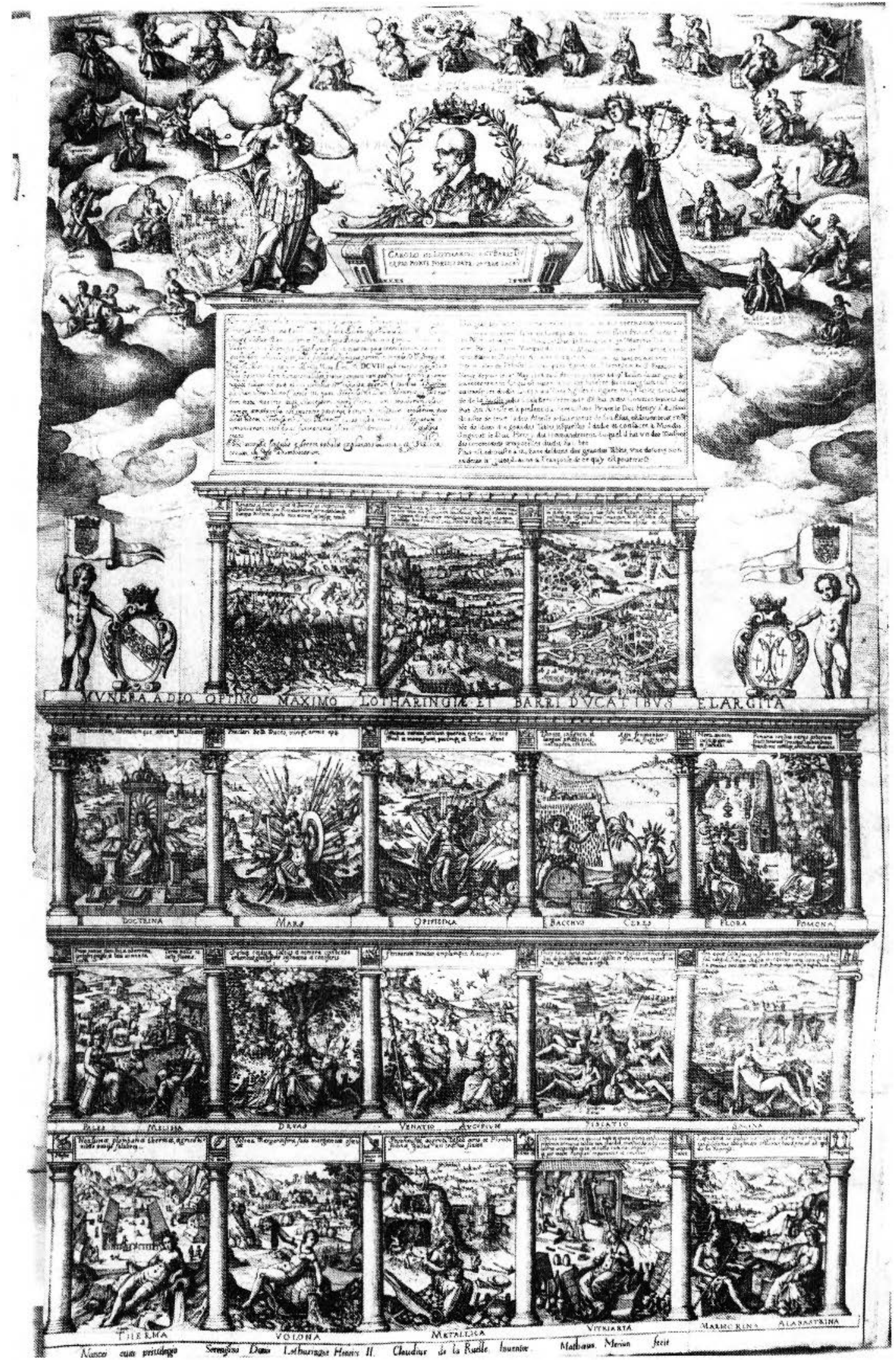

Figure 1. Pompe funèbre du duc Charles III, frontispice. Eau-forte par Matthieu Mérian d'après Claude de La Ruelle. 625 × $385 \mathrm{~mm}$. Bibliothèque municipale de Nancy (cliché Ph. Pillot, Scrvice photographique de la Bibliothèque Interuniversitaire, Nancy). 


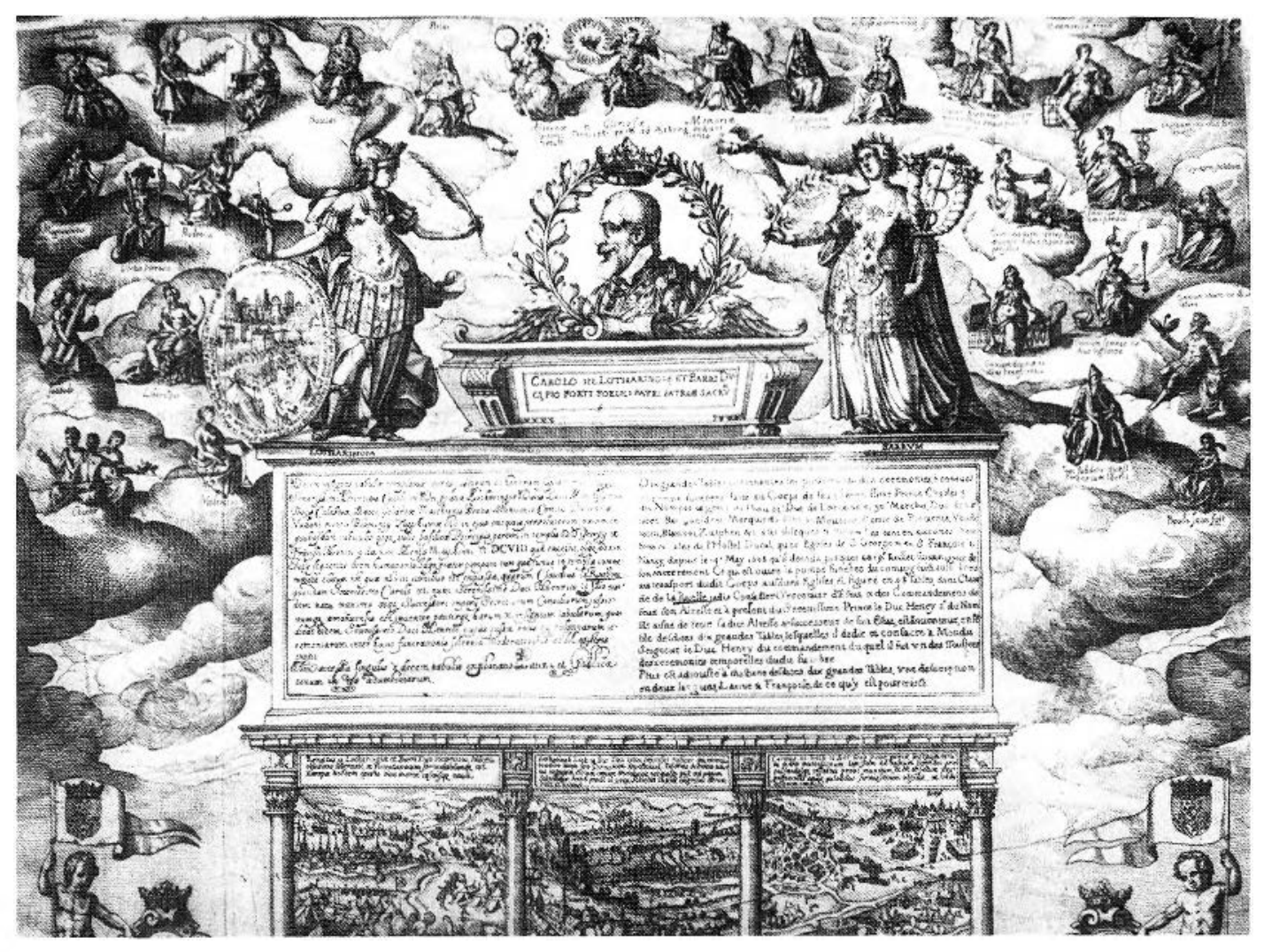

Figurf. 2. Détail : les allégories de la prospérité lorraine.

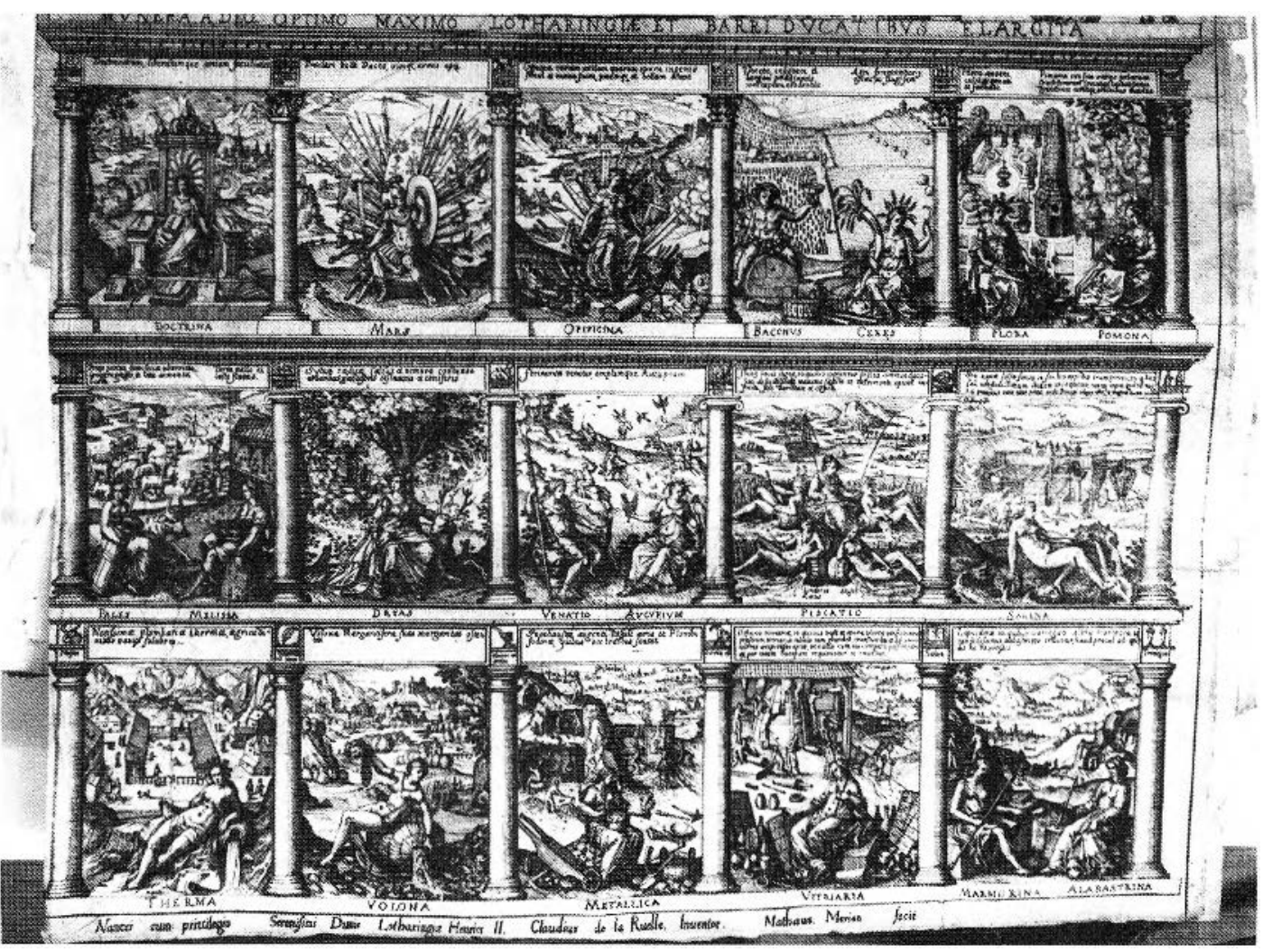

Figure 3. I ces allégories des vertus et mérites de Charles ni. 


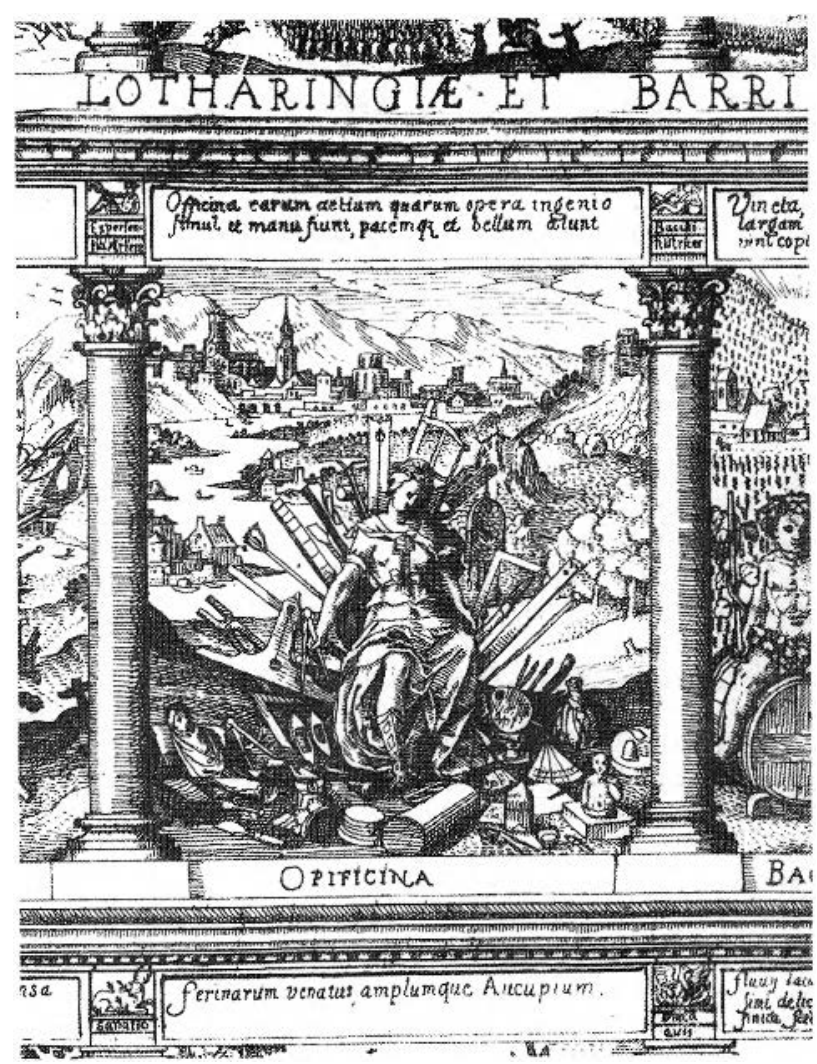

Figl'Re 4. Opificina.

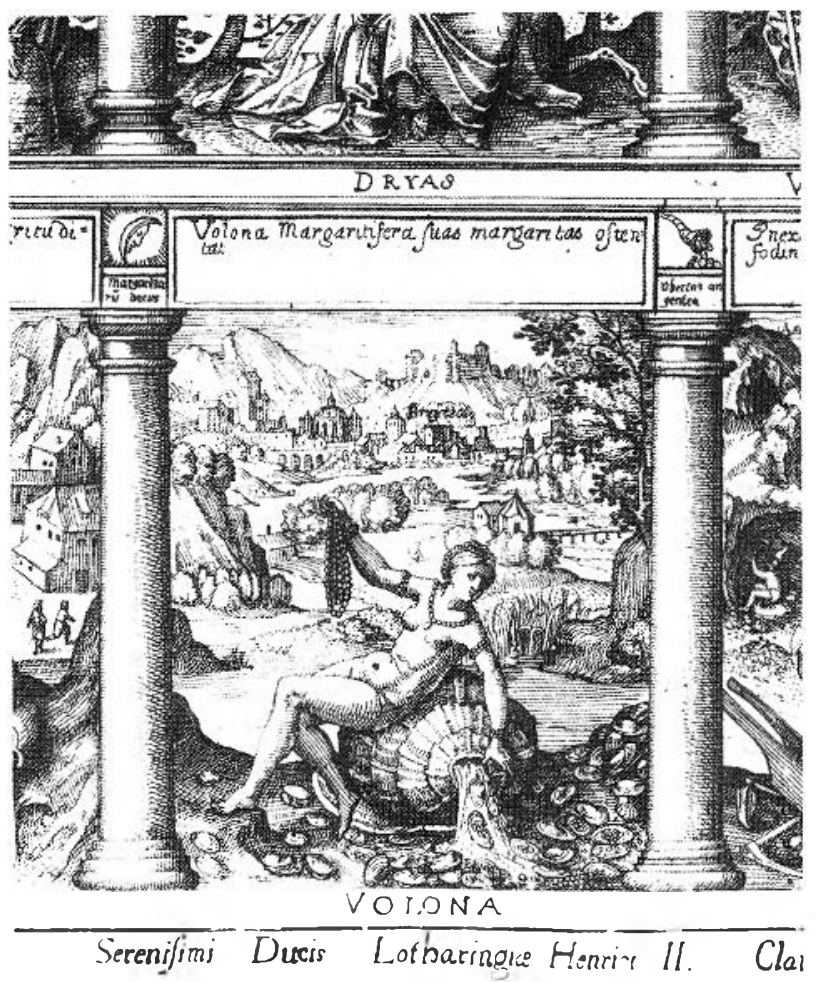

Figl Re 5. Volona (la Vologne et ses huîtres perlières). A l'arrière-plan, la ville de Bruyères (Vosges). 


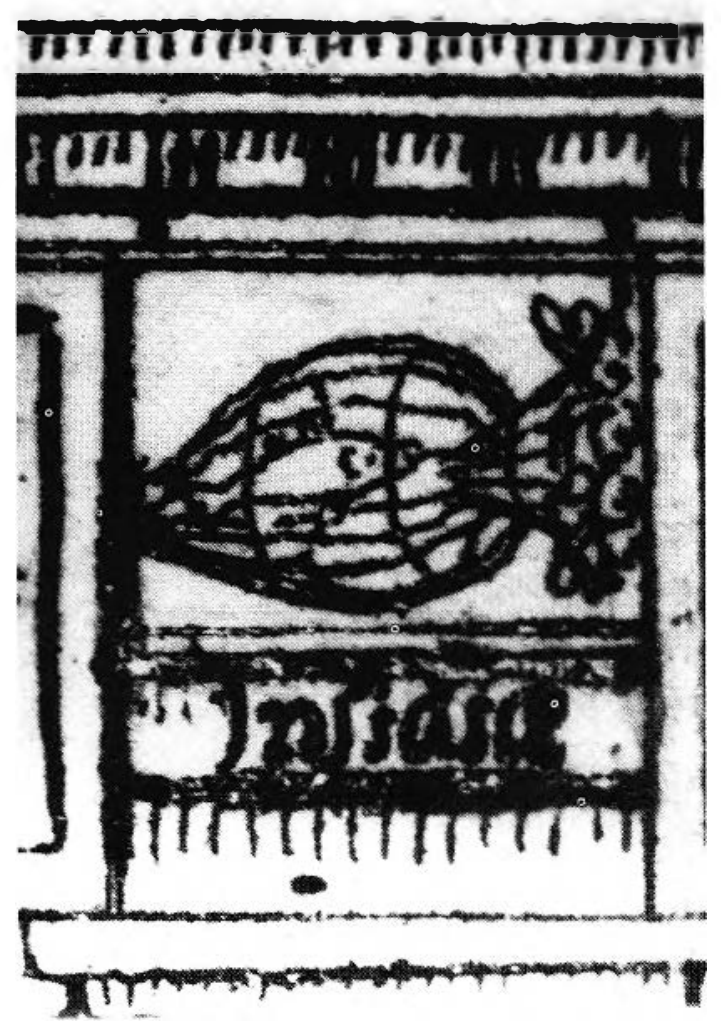

Figure 6. Emblème de l'architrave, $9 \times 6 \mathrm{~mm}$ : Insidiae.

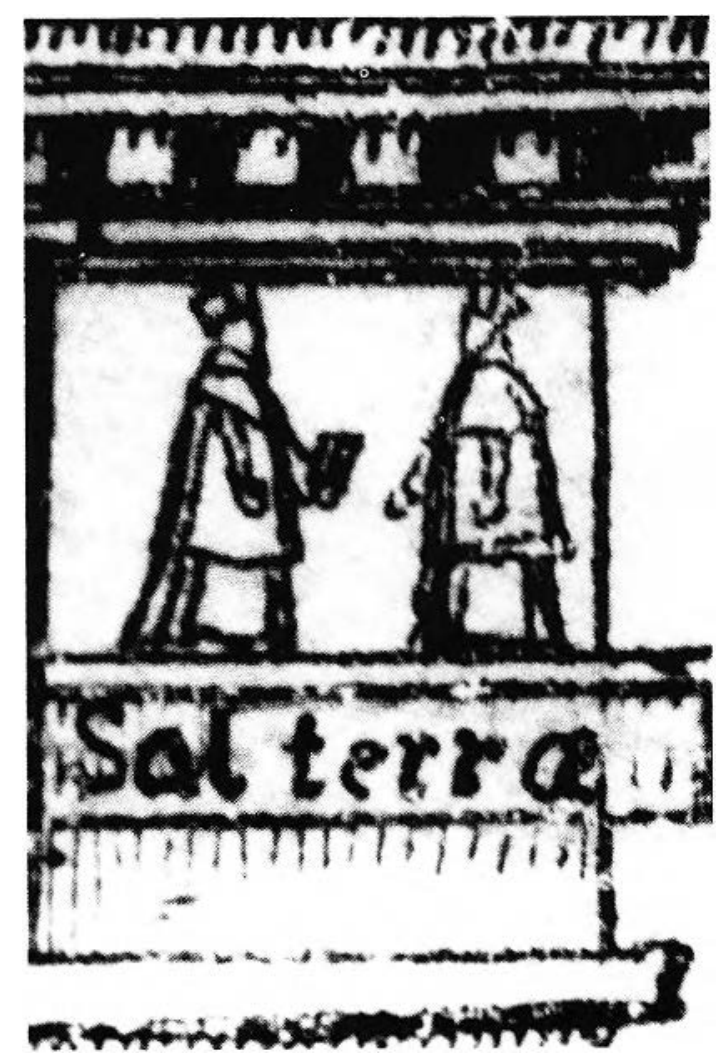

Figure 8. Emblème de l'architrave: Sal terrae.

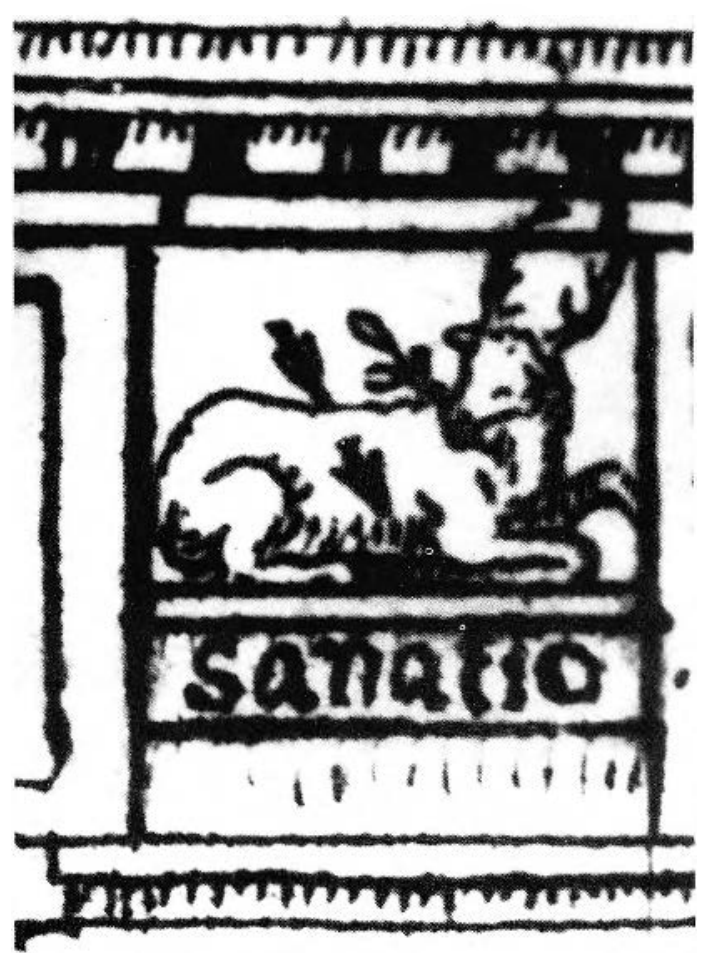

Figure 7. Emblème de l'architrave: Sanatio.

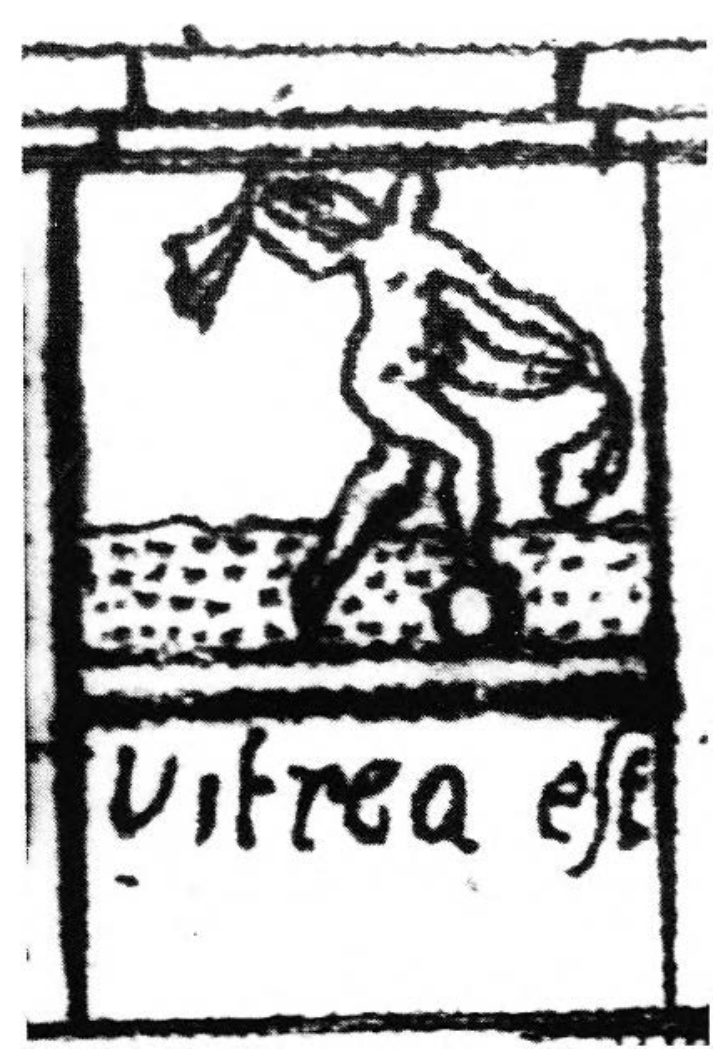

Figure 9. Emblème de l'architrave: Vitrea est. 


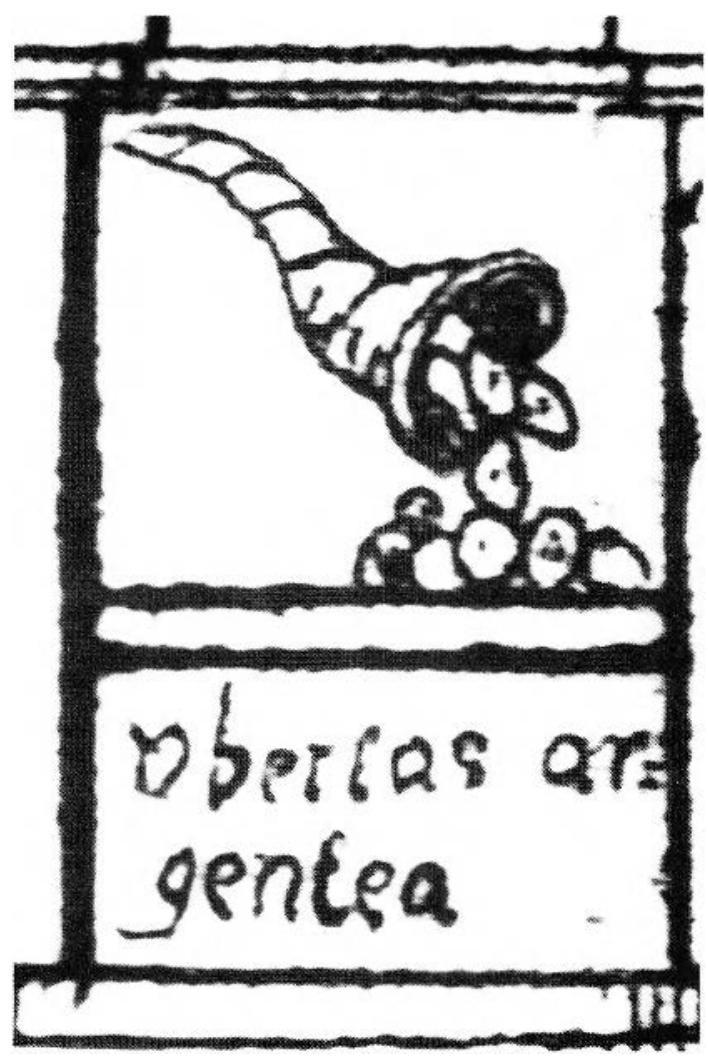

Figure 10. Emblème de l'architrave: Ubertas argentae.

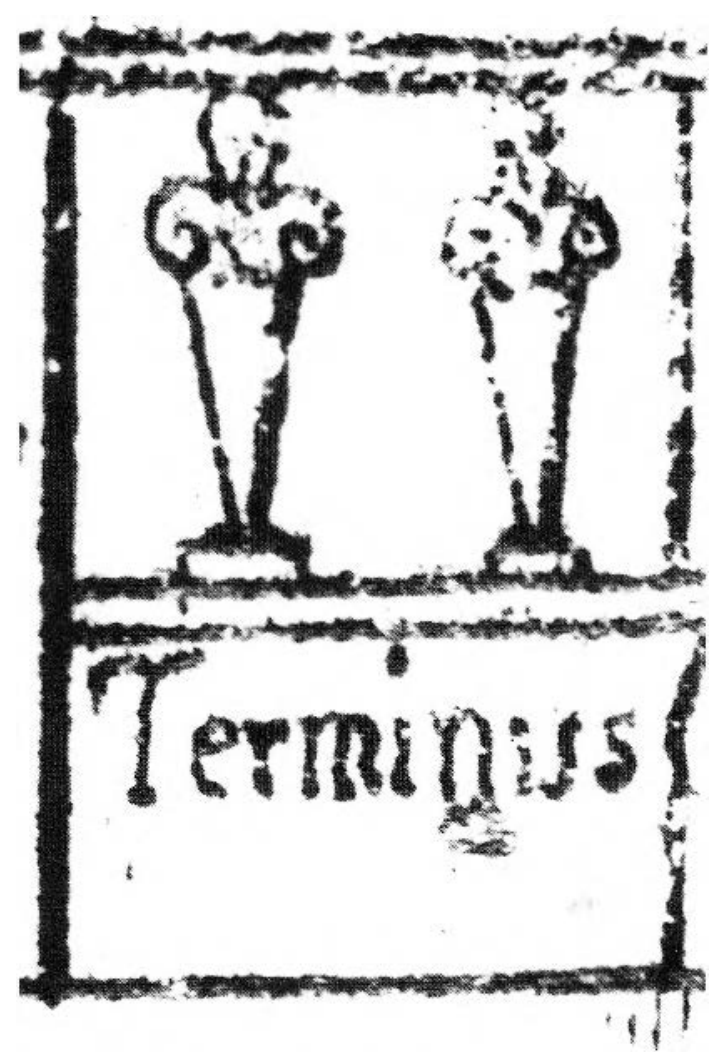

Figure 11. Emblème de l'architrave: Terminus.

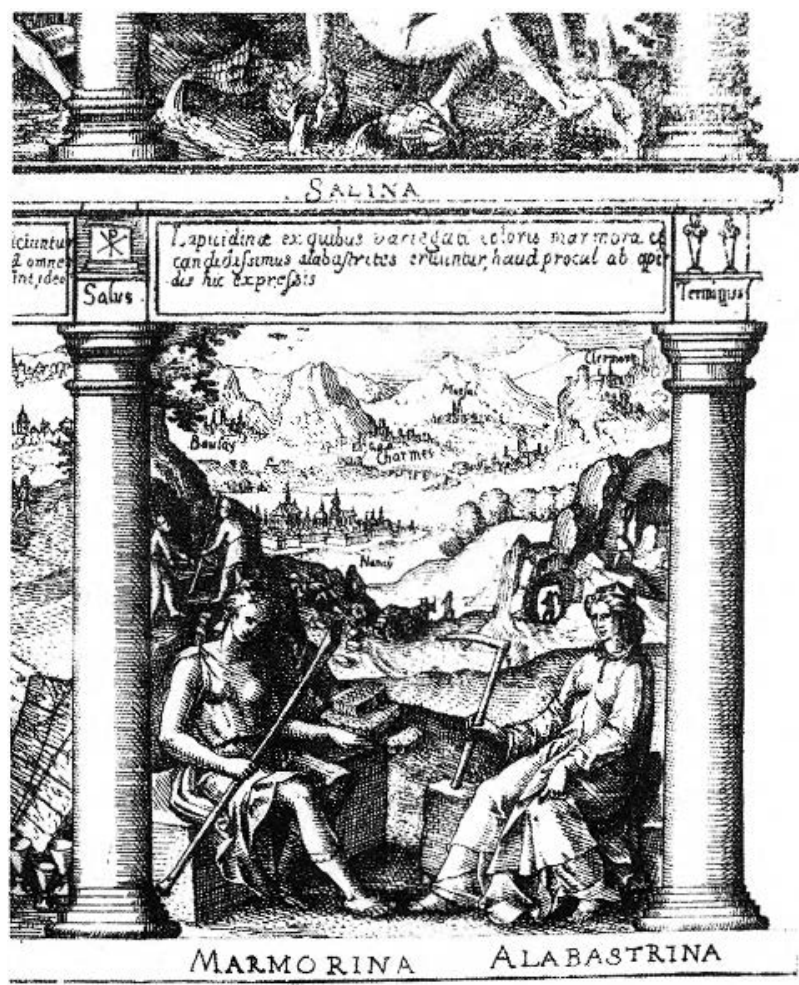

Figurf. 12. Marmorina Alabastrina (à l'arrière-plan, Nancy, Boulay, Charmes, Marsal, Clermont). 